\title{
LAS CITAS EN LA COMUNICACIÓN ACADÉMICA ESCRITA ${ }^{1}$
}

\author{
María Palmira Massi \\ Universidad Nacional del Comahue, Argentina
}

\section{INTRODUCCIÓN}

El discurso académico presenta la discusión sobre un aparato conceptual de una forma rigurosamente elaborada que exige la observancia de normas convenidas en el proceso de composición. La heurística de este género discursivo parte de preguntas y, a través de la descripción, la clasificación y la explicación, culmina en la construcción de sistemas de ideas dirigidas a la comunidad científica. El discurso académico se estructura en función de la intención persuasiva del autor, por tanto la estructura retórica predominante gira en torno a la organización de argumentos y a los modos de presentarlos. Su elaboración requiere de la revisión de las fuentes bibliográficas ya que se orienta a la transmisión y producción de conocimientos (Elejalde 1997).

En el contexto universitario, la construcción de tal discurso confronta al estudiante - de grado o de postgrado - con un considerable desafío, pues debe cumplimentar una serie de exigencias teóricas y metodológicas impuestas por la cátedra y la institución. En un escenario ideal, la formación del futuro profesional debería proporcionar el instrumental necesario para desarrollar tal tarea, aunque la realidad - al menos en Argentina - parece revelar que es preciso implementar su enseñanza formal en el ámbito universitario $^{2}$. En tal sentido, el presente trabajo forma parte de una programa pedagógico que aborda el análisis de textos académicos etiquetados como tales en las observaciones explícitamente verbalizadas por los usuarios que normativizan su retórica (docentes, investigadores, currículas, instructivos y manuales). La observación empírica y el análisis de tales textos constituyen el punto de partida hacia la detección de los instrumentos necesarios para satisfacer necesidades pragmáticas en el contexto académico. Los objetivos de nuestro proyecto de investigación comprenden: 1. el análisis de los instructivos circulantes para la realización de monografías o papers; 2. el estudio de los diferentes pasos o procedimientos lingüísticos en la elaboración de trabajos académicos; 3. el diseño de nuevos instructivos que contemplen la composición como proceso a los efectos de facilitar la enseñanza formal en el ámbito universitario.

En este artículo nos proponemos analizar una de las etapas de este proceso, la inclusión de procedimientos de cita, y desmontar las técnicas más frecuentes y efectivas en la formulación lingüística. En efecto, la referencia al conocimiento pre-existente constituye la 'materia prima' en la elaboración de trabajos académicos, pues cumple la función de legitimar tal conocimiento como así también de reforzar y afirmar la

\footnotetext{
${ }^{1}$ Este trabajo forma parte del Proyecto de investigación Hacia una retórica del discurso académico, actualmente en ejecución en la Facultad de Derecho y Ciencias Sociales (FADECS) - Escuela Superior de Idiomas (ESUI). Universidad Nacional del Comahue. Argentina. El proyecto fue aprobado por la Secretaría de Investigación de esta universidad.

${ }^{2}$ Resulta alentador el creciente interés por el estudio del discurso académico en Latinoamérica, tal como lo corroboran los valiosos aportes teóricos y metodológicos de Parodi Sweis 1999, Martínez 2001a, 2001b, 2002, Carlino et. al. 2004, y de numerosos investigadores de la Cátedra UNESCO de Lectura y Escritura en América Latina. En Argentina, se observan diferentes líneas de investigación muy relacionadas entre sí, aunque demarcadas por perspectivas teóricas y modalidades diversas. Algunos estudios versan sobre el desarrollo de habilidades para lograr la comprensión y producción de textos académicos, otros se focalizan en las partes constitutivas de formatos específicos desde perspectivas funcionales, mientras que otros realizan análisis de aspectos tales como el léxico, la modalidad o la selección temática.
} 
argumentación. La apelación a la autoridad otorga solidez al trabajo ya que permite corroborar que se ha investigado un tema en profundidad y contribuye a documentar el propio nivel científico y educativo.

\section{CORPUS Y METODOLOGÍA DEL ESTUDIO}

El corpus de trabajo consta de insumos - artículos, ensayos e informes en soporte electrónico utilizados en la discusión de un tema proveniente del área de las Ciencias Sociales - globalización - con el objetivo de proporcionar contenidos que orienten al alumno en la formación de opinión. El fin último es proporcionar contenido conceptual para la elaboración de una monografía de compilación ${ }^{3}$, que hemos definido como un texto de carácter informativo-referencial que presenta dos características esenciales: (1). su objeto es concreto y limitado, ya que comprende la síntesis del relevamiento bibliográfico e intenta dar una visión panorámica acerca de un determinado tópico; (2). tal objeto es descripto en forma exhaustiva, con una sólida organización, una adecuada selección bibliográfica y el montaje de un aparato crítico que otorgue las necesarias referencias a la obra.

En este contexto, el sistema de reproducción de otros discursos comprende la cita textual, la alusión a otros textos y las citas ideológicas que se realizan mediante paráfrasis o resúmenes de la opinión de otros autores. Con una metodología cualitativa (Denzin y Lincoln 1994) y desde una perspectiva retórica (Perelman 1979, Perelman y Olbrechts-Tyteca 1989, Albaladejo Mayordomo 1988) ${ }^{4}$, se aborda entonces la instancia de puesta en discurso - elocutio - y se investiga las variantes de las citas y el contexto argumentativo en el que están inmersas a los efectos de determinar su función en el texto. Asimismo, se discuten las diferencias en los modos de negociar y establecer consensos con los representantes de las comunidades académicas a las que se alude en forma explícita.

\section{MECANISMOS DE INCORPORACIÓN DE LA CITA EN EL DISCURSO ACADÉMICO}

La cita es la reproducción de otro discurso, un aspecto o una parte del mismo, en el propio (Reyes 1993). En la elaboración de una monografía compilativa, el objetivo central es la consideración meticulosa de los referentes bibliográficos de que se dispone y su comparación y evaluación con vistas a una toma de posición por parte del escritor a la luz de los conceptos analizados. A fin de reproducir el pensamiento y las ideas ajenas, los procedimientos más frecuentes que se observan en el corpus son: (1). la repetición de las mismas palabras empleadas en el discurso de origen - estilo directo - (2). la modificación de la forma del discurso citado, a partir del cambio de algunas marcas de enunciación, sin alterar el contenido - estilo indirecto - con sus respectivas variantes. El estilo directo - que concede al enunciado el carácter de literalidad - presenta los siguientes mecanismos dispositivos.

\footnotetext{
${ }^{3}$ Las modalidades que se abordan en el Taller de Escritura implementado por el proyecto de investigación son las siguientes: la monografía compilativa, que se basa en el relevamiento bibliográfico y comprende la definición del objeto de estudio, la elaboración de síntesis e informes de lectura, y culmina con el posicionamiento crítico del autor; la monografía informe, que trabaja con datos, estadísticas u observaciones empíricas; la monografía de investigación, que exige un sistema sólido de pruebas y conclusiones proyectos de investigación, tesinas, tesis, artículos científicos, reseñas de libros, artículos de divulgación y ponencias en congresos -, y la monografía ensayo, un género más libre que prescinde de la demostración y la documentación.

${ }^{4}$ La perspectiva retórica estudia la estructura de la argumentación en textos escritos y considera las tres operaciones constituyentes de cualquier tipo de discurso: inventio, dispositio y elocutio, esto es, la selección de insumos, la disposición sintagmática en un texto y la selección de recursos eficaces en su formulación lingüística (Perelman 1979, Perelman y Olbrechts-Tyteca 1989). Esta línea teórica se complementa con los aportes de la retórica textual, que propone una metodología de Análisis del Discurso a partir de la concepción del texto como un dispositivo retórico e incorpora categorías analíticas de disciplinas afines como la semiótica y la pragmática (Albaladejo Mayordomo 1988).
} 
(a). Directo antepuesto: el discurso referido encabeza el enunciado con un verbo de comunicación que alude al discurso precedente. También es frecuente incorporar la cita textual inmediatamente seguida por la fuente de referencia.

(1). "La globalización no es sólo económica y financiera. Quien crea esto se equivoca enormemente. La globalización cambia el estilo de vida, la tradición y las costumbres, tiene un impacto sobre las relaciones personales y familiares, y afecta también a la ciencia y la tecnología", dice Anthony Giddens, director de la London School of Economics, teórico e inspirador del nuevo laborismo inglés.

(2). "Antes lo local, lo nacional, lo internacional se representaban como escalones impermeables el uno al otro, compartimentados. En nuevo esquema de representación de la empresa y del mundo en el que opera, como red, propone un modelo de interacción entre estos tres niveles" (Mattelart 1985).

(b). Directo pospuesto: en la estructura del enunciado se observa un verbo de comunicación o una expresión introductoria que precede la cita textual.

(3). El Fondo Monetario Internacional define la globalización como "la interdependencia económica creciente en el conjunto de los países del mundo, provocada por el aumento del volumen y de la variedad de las transacciones transfronterizas de bienes y servicios, así como de los flujos internacionales de capitales, al mismo tiempo que por la difusión acelerada y generalizada de la Tecnología" (Citado en Wolf, 1997:14).

(4). Desde la tradición del pensamiento crítico latinoamericano, Pablo González Casanova intenta recuperar algunas dimensiones poco socorridas en este debate y propone "pensar que la globalización es un proceso de dominación y apropiación del mundo".

(c). Directo discontinuo: la cita textual se descompone en dos partes: una parte precede al verbo declarativo y la otra le sigue.

(5). "En consecuencia - mantiene Zubero - un enfoque crítico debe rechazar de forma categórica todo intento de presentar la tecnología como neutral, como una simple herramienta, cuyos efectos dependerán sólo del uso que de la misma se haga, tal y como pretende el enfoque capitalista...".

El estilo indirecto abarca, en un sentido genérico, cualquier procedimiento de cita no literal. El contenido del discurso referido en esta modalidad puede coincidir total o parcialmente con el del discurso original. Las variantes más utilizadas se detallan a continuación.

(a). Indirecto genuino: no se afecta el contenido del discurso referido, sino sólo su enunciación; se introduce con un verbo de comunicación y un nexo del tipo de que o como.

(6). El investigador mexicano Raúl Trejo define la globalización como la omnipresencia, totalizadora y envolvente, de intercambios y recursos que a menudo hacen creer que el mundo es uno sólo.

(7). Por su parte, Noam Chomsky asegura que la necesidad expansionista de la sociedad burguesa se conceptualiza actualmente como globalización, extensión a todo el globo, basada en la información y los multimedia.

(8). El comunicólogo Jesús Martín Barbero sostiene que global es el espacio/tiempo que necesitan y producen el mercado y las nuevas tecnologías.

(9). Lineares y Ortiz (1995) consideran como características principales de la sociedad de la información las siguientes; la terciarización, la automatización, la globalización, la información como materia prima, la interactividad y la complejidad.

(b). Indirecto con segmentos textuales: se resume o sintetiza lo dicho a través del recurso de la

paráfrasis, pero además se transcriben textualmente algunas expresiones a las que se le asigna relevancia por medio del encomillado. Esta marca tipográfica es obligatoria y su ausencia podría hacer que el autor incurra en el plagio.

(10). Como apuntó el sociólogo británico y director de la London School of Economics, Anthony Giddens, globalización es un término que, usado con tanta frecuencia, sin embargo, está muy pobremente conceptualizado. La orientación analítica y la disposición ideológica separa entre "hiperglobalizadores" y "escépticos de la globalización" (Giddens 1996).

(11) Podríamos coincidir con Giddens, quien sugiere que "elaborar una conceptualización adecuada de este fenómeno debe diferir de ambos enfoques", y debe ponerse atención en varias cuestiones: esta sacudida de la sociedad mundial tiene numerosas causas y no una sola... 
(c). Indirecto narrativizado: se refiere lo dicho en forma sintética o resumida.

(12). Entre los primeros - hiperglobalizadores -, ligados sobre todo al ambiente de los negocios y con gran influencia en las elites económicas y políticas, la globalización se entiende como la expansión del mercado a escala mundial. Dentro de esta corriente, Kenichi Ahmae argumenta que en el futuro la nueva economía mundial tendrá como núcleo no a los estados-nación sino a muchas regiones entrelazadas, a modos de estados -región, ciudades -Estado o ciudades -globales.

Tanto en el discurso directo como en el indirecto, se incorpora una masa textual proferida por otro o su esencia en forma de resumen o compendio - al flujo de la composición propia. La perspectiva retórica subraya la importancia del contexto comunicativo, entendido como el equilibrio entre las exigencias del escritor, las expectativas del destinatario, la complejidad del tema y la finalidad suasoria del texto. En nuestro corpus, la cita permite reconstruir el aparato bibliográfico relevado por el investigador y su posicionamiento epistemológico. Además, contribuye a configurar textualmente su pertenencia ideológica y orienta al lector acerca de la misma.

En otros contextos, por ejemplo el discurso periodístico, el político o el autobiográfico ${ }^{5}$, una estrategia frecuente consiste en extraer una emisión del contexto en el que fue proferida e introducirla en el propio discurso a veces sin marcas gramaticales explícitas, mediante alusiones, entonación o, simplemente, la utilización de léxico que se adjudica a otras personas. A diferencia de estos formatos, el discurso académico construye su línea argumentativa sobre la base de ideas y razonamientos previos, que le sirven de punto de partida. En efecto, la introducción de citas en este contexto responde a la normativa de hacer referencia a la voz de expertos en un área determinada, a cuya posición se le asigna un valor por la profundidad o la seriedad del trabajo realizado. En este sentido, la finalidad es orientar al lector en la discusión de un tema particular, para lo cual es menester que el investigador conozca la bibliografía existente y pueda entablar un diálogo con la misma. En función de la alta convencionalidad de las normas de estilo que deben observarse en este género, un rasgo distintivo del discurso académico es la alta densidad de citas expresas y la considerable extensión que éstas ocupan en el texto.

\section{VERBOS INTRODUCTORIOS DEL DISCURSO REFERIDO}

En el texto académico, el procedimiento canónico de inclusión de citas se realiza a través de verbos de decir, también denominados verbos de comunicación o verba dicendi (Maldonado 1991:41), que significan la expresión de un pensamiento o una idea. Estos se utilizan indistintamente tanto en la introducción del estilo directo como del indirecto. Los verbos introductorios más frecuentes dentro de esta categoría, además de decir - el más general de este grupo semántico - son los siguientes: referir, comentar, explicar, afirmar, asegurar, preguntar, contestar, responder, entre otros. Se observa, además, una alta densidad de verbos de opinión (Maldonado 1991:58) que describen acciones que pueden realizarse con palabras. Los que predominan en nuestro corpus son: opinar, considerar, apuntar, postular, sugerir, sostener, mantener, manifestar, puntualizar, argumentar. El verbo pensar, catalogado como verbo de pensamiento y percepción por Reyes 1993:19, permite transmitir contenidos y tiene alguna recurrencia aunque baja - en el corpus. También son frecuentes los verbos que no especifican una comunicación verbal, del tipo de definir, demostrar, proponer, probar, indicar y las expresiones introductorias de discurso referido $^{6}$ - según $X$, tal como plantea $X$, para $X$, entre otras -. En función del valor temporal, predominan los

\footnotetext{
${ }^{5}$ Un análisis crítico de la incorporación de voces en la producción de prácticas discursivas en los medios de comunicación se ofrece en Massi 2001, 2005.

${ }^{6}$ Reyes 1994:41 sostiene que estas expresiones cumplen las funciones de los evidenciales citativos, pues sirven para señalar que el origen de la aserción que sigue pertenece a otro locutor.
} 
verbos retrospectivos - remarcar, recalcar, subrayar, recordar, repetir, objetar, aclarar, precisar - ya que los fragmentos citados hacen referencia a textos anteriores o contemporáneos y aluden a la fuente de un conocimiento u opinión.

En el cuadro a continuación se presentan las modalidades introductorias de citas que aparecen con mayor frecuencia en el corpus relevado.

\section{Modalidades de introducción de discurso referido}

- verbos de decir: referir, comentar, explicar, afirmar, asegurar, preguntar, contestar, responder, entre otros.

- verbos de opinión: opinar, considerar, apuntar, postular, sugerir, sostener, mantener, manifestar, puntualizar, argumentar.

- verbos que no especifican una comunicación verbal: definir, demos trar, proponer, probar, indicar.

- expresiones introductorias: según X, tal como plantea X, para X, entre otras.

- verbos retrospectivos: remarcar, recalcar, subrayar, recordar, repetir, objetar, aclarar, precisar.

Como puede observarse, la naturaleza de estos nexos es relativamente neutra, si se la compara con la de los verbos que se utilizan en otros formatos o modalidades discursivas ${ }^{7}$. Sin embargo, algunos de los verbos reseñados aportan distintos matices de significado acerca del acto lingüístico. Por ejemplo, tanto afirmar como asegurar indican el grado de certeza con que se expresa el discurso citado; en aclarar, precisar y declarar subyace la intención de hacer más claro y comprensible el discurso referido. Algunos verbos implican la verdad o la falsedad del discurso citado - Cf. demostrar e hipotetizar - mientras que otros conllevan una valoración negativa - refutar, criticar -. Otros hacen hincapié en lo novedoso y original de la cita - e.g. revelar - mientras que otros nexos sitúan la nueva masa textual en una orientación argumentativa - responder, advertir, repetir, concluir -. De este modo, el verbo introductorio seleccionado incluye información que, a veces, condiciona la interpretación de la cita e impone una determinada lectura por parte del destinatario del texto.

\section{CONCLUSIONES}

La cita constituye un fenómeno constante en el discurso académico pues es el punto de partida para la construcción del objeto. A modo de síntesis, resumimos aquí las funciones predominantes: (1). recuperar los conceptos vertidos por la voz experta en el área que se investiga por su relevancia y pertinencia; (2). indicar y documentar la procedencia del conocimiento; (3). establecer relaciones entre un texto existente y el que está en elaboración con la finalidad de comentar, asentir, disentir o señalar incongruencias.

La inclusión de citas requiere la creación de un contexto propicio a los efectos de lograr su integración con el nuevo texto. A tal fin, el investigador selecciona los aspectos que considera relevantes para respaldar una finalidad argumentativa concreta que elabora de antemano. Al extraer un material que ya tiene su significado en un discurso, realiza los ajustes y transformaciones correspondientes para hacerlo funcionar en un nuevo sistema de significación. De este modo, las palabras o ideas de otros se funden o integran a las propias en una suerte de diálogo creativo. El producto resultante constituye una simbiosis de argumentos propios y ajenos, con una síntesis de la posición alcanzada en base al razonamiento previo. Una estrategia frecuente consiste en introducir una cita o un conjunto de citas que adscriben a una determinada corriente epistemológica para luego proceder a una evaluación minuciosa que permita establecer puntos de consenso o disenso con el posicionamiento propio respecto del tema en cuestión. 
También se puede introducir una cita y confrontarla con otra(s) para generar una situación polémica, realizar un análisis a fin de inclinarse por una o varias, desechar otra(s) y proceder a la fundamentación. Estos constituyen diferentes modos de negociar y establecer grados de pertenencia con las comunidades académicas que se mencionan. A través de los procedimientos señalados, se produce una 'imbricación' de discursos: el conocimiento referido se integra en un discurso sobre la realidad al tiempo que permite al autor hacer suya la voz citada y fundamentar la posición teórica - e ideológica - que orienta su texto.

\section{REFERENCIAS BIBLIOGRÁFICAS}

AlbalAdEJo MAYORDOMO Tomás. Semántica y sintaxis del texto retórico: inventio, dispositio y partes orationis. Revista Estudios de Lingüística. Universidad de Alicante. España. 1988.

CARLINO Paula (coord.). Textos en contexto: Leer y escribir en la universidad. Buenos Aires: Asociación Internacional de Lectura. 2004.

CARRANZA Isolda. Argumentatividad y variedad de formas de discurso referido en narraciones conversacionales. Actas del I Simposio Internacional de Análisis del Discurso. Universidad Complutense de Madrid y Université de Paris XIII. Madrid. España. 1998.

DENZIN Norman y Y.S. LINCOLN Introduction: entering the field of qualitative research, en DENZIN Norman y Y.S. LINCOLN (eds.). Handbook of Qualitative Research. California: Sage Publications. 1994.

ELEJALDE Alfredo. El discurso académico. http://macareo.pucp.edu.pe/ elejalde/ensayo. 1997.

FORCADA Miguel. ¿Cómo se escriben y publican trabajos de investigación?. Departament de Llenguatges $i$ Sistemes Informàics. Universitat d’Alacant. España. 2001.

MALDONADO Concepción. Discurso directo y discurso indirecto. Madrid: Taurus Universitaria. 1991.

MARTÍNEZ María Cristina (ed. y comp.). Comprensión y producción de textos académicos: expositivos y argumentativos. Universidad del Valle. Cali. Colombia: Editorial Taller Artes Gráficas. 2001a.

MARTínez Solís María Cristina. Análisis del discurso y práctica pedagógica. Una propuesta para leer, escribir y aprender mejor. Buenos Aires: Editorial Homo Sapiens. 2001b.

MARTÍNEZ María Cristina. Estrategias de lectura y escritura de textos. Perspectivas teóricas y talleres. Universidad del Calle. Cali. Colombia: Editorial Taller Artes Gráficas. 2002.

MASSI María Palmira. Estrategias de incorporación de voces en la entrevista autobiográfica. Presentación en el II Coloquio Nacional de Investigadores en Estudios del Discurso: "El estudio del discurso: metodología multidisciplinaria". La Plata. Argentina. 2001.

MASSI María Palmira. Negar, rectificar, ironizar... Otras formas de la polifonía. Análisis de la producción de prácticas discursivas en los medios de comunicación. Revista de la Facultad de Derecho y Ciencias Sociales. Vol. 11. Universidad Nacional del Comahue. Argentina (en prensa). 2005.

PARODI SWEIS Giovanni. Relaciones entre lectura y escritura: una perspectiva cognitiva discursiva. Bases teóricas y antecedentes empíricos. Valparaíso: Ediciones Universitarias de Valparaíso de la Universidad Católica de Valparaíso. 1999.

\footnotetext{
${ }^{7}$ Carranza 1998 explora las formas de discurso referido en narraciones conversacionales; Massi 2001, 2005 analiza la incorporación de voces en la entrevista autobiográfica televisiva.
} 
PERELMAN Chaï m. The new rhetorics and the humanities. Londres: Reidel Publishing Co. 1979.

PeReLMAN Chaï m y Lucie OlBRECHT-TYTECA. Tratado de la argumentación. La nueva retórica. Madrid: Gredos. 1989.

REYES Graciela. Los procedimientos de cita: estilo directo y estilo indirecto. Madrid: Arco Libros. 1993.

REYES Graciela. Los procedimientos de cita: citas encubiertas y ecos. Madrid: Arco Libros. 1994.

CORPUS

CARBonell A. "La globalización nos envuelve". Escuela de Comunicación Social y Periodismo, Universidad Sergio Arboleda. 2002.

GANDARILLA SALGADO J. “¿De qué hablamos cuando hablamos de la globalización?”. Revista Herramienta. http://www.rebelion.org/sociales/gandarilla260602.htm. 2002.

GIDDENS A. "Efectos de la globalización”. Diario El Mundo. España (17/07/02). 2002.

RAGHA VAN C. “¿Qué es la globalización?”. Revista del Sur63/64. 1997.

SANTAMARTA J. “¿Qué es la globalización?”. Iniciativa Socialista. http://www.plades.org.pe/etextos/. 2002. 


\title{
Contactar
}

Revista lberoamericana de Educación

\author{
Principal OEI
}

